\title{
Commissioning and performance of the CMS silicon microstrip detector
}

\author{
Regina Demina ${ }^{1}$ \\ University of Rochester \\ Rochester, NY, USA \\ E-mail: demina@fnal.gov
}

\section{On behalf of CMS Collaboration}

The CMS silicon strip tracker is the largest device of this type ever built for detection of charged particles produced in beam-beam collisions. There are 24244 single-sided micro-strip sensors covering an active area of over 200 square meters, and nearly 10 million channels to be read out. The detector was installed inside CMS in December 2007, and it was commissioned during the summer 2008. Since then a large quantity of cosmic ray data has been collected and its performance have been assessed in combined operation with all other CMS sub-detectors. The commissioning strategy, the operational experience acquired during the data taking period, and the detector performance results are reported in this paper.

9th International Conference on Large Scale Applications and Radiation Hardness of Semiconductor Detectors-Rd09

Florence, Italy 30 September - 2 October 2009

Speaker 


\section{Introduction}

In 1999 the CMS collaboration reached a bold decision to build an all silicon tracking (SiTrk) device. The addendum to TDR [1] called for a system with almost 10 million readout channel with a total silicon surface area of over $200 \mathrm{~m}^{2}$. At that time the largest system built (D0 SMT [2]) had a bit less than 1 million channels. To meet this goal and ensure a robust operation of the system for 10 years choices were made to simplify the design as much as possible, but not more than that. Single sided low resistivity silicon sensors presented a sensible option, which was well understood at that time. CMS silicon tracker consists of the outer barrel (TOB), inner barrel (TIB), inner disks (TID) and endcap disks (TEC). The TOB and the outer regions of the TEC are built using 500 $\mu \mathrm{m}$ thick silicon sensors, while the sensors in the TIB, the TID and inner parts of the TEC are $300 \mu \mathrm{m}$ thick. An analogue readout chip called APV25 was specifically developed for the CMS SiTrk using $0.25 \mu \mathrm{m}$ technology[3]. The electrical signal is then converted into an optical one and sent for digitization outside of the detector volume via optical fibers. The entire detector volume of $24.4 \mathrm{~m}^{3}$ is expected to be operated at a temperature of $-20{ }^{\circ} \mathrm{C}$.

A large number of industrial enterprises were involved in the production of the components for the system. The production was distributed over many module assembly/testing/bonding and integration facilities around Europe and the USA. With such a complex production logistics, the quality assurance was of the utmost importance. By the end of summer 2006 all the components were delivered to CERN and the complex process of integration and commissioning of the tracker system began. The SiTrk went through several important steps in the detector commissioning and performance studies. In August-September 2006 several modules were installed inside CMS and their performance in magnetic field was tested in the so called Magnet Test Cosmic Challenge (MTCC)[4]. Though some of the components were not final, the MTCC was essential in establishing the tracker operation within the global CMS readout scheme and set important benchmarks for the detector performance. In the mean time the standalone performance of about $20 \%$ of the tracker was tested using cosmic rays triggered on by plastic scintillators. Basic measurements of the signal to noise ratio, cluster width and position resolution were done using standalone tracker data, while first attempts to measure the Lorentz angle were made with the MTCC data. On December 16, 2007 the tracker was moved from its assembly facility to LHC Point 5, lowered into the collision hall and inserted into the CMS detector. Since then, several extended runs were taken with and without magnetic field, mainly using the CMS muon system for triggering. The angular distribution of the cosmic tracks reaching CMS deep under ground did not provide a uniform illumination of the tracker system; however these data allowed to perform a rather detailed characterization of the system performance as well as to achieve a fairly good alignment. The results presented in these proceedings were primarily obtained during cosmic data taking with a 3.8T magnetic field, during October-November, 2008, the so called Cosmic Run At Four Tesla (CRAFT08). In summer 2009 more data were taken with magnetic field, but these data are still being analyzed and we only report on them briefly. 
During the CRAFT08 run the tracker took part in about $90 \%$ of the global runs. The fraction of the operational channels varied depending on the detector subsystem, but it was always above $95 \%$ : more precisely it was $96.7 \%$ for the TIB/TID, $98.3 \%$ for the TOB, $99.2 \%$ for the TEC+, and $98.3 \%$ for the TEC-.

\section{Signal to noise ratio}

The signal-to-noise ratio is an important parameter for a reliable tracker operation. This ratio was measured using CRAFT08 data, during which the detector was operated in the so-called peak readout mode of the front end APV25 chip. For this purpose the tracking was performed using cosmic muon events, and only clusters attached to tracks were used in the definition of the signal-to-noise ratio. The charge collected from the silicon sensors was corrected for the track inclination angle. Fluctuation in energy deposition in thin layers is best described by straggling functions[5], yet for the purpose of signal-tonoise determination we use Landau distribution. Fluctuations of the APV25 readout can be described by Gaussian distribution[3]. Cluster noise was defined as a sum of strip noise, measured for the respective strips during pedestal runs. The distribution used to describe the shape of the signal to noise ratio from silicon sensors was Landau convoluted with Gaussian, as shown in Fig.1. The most probable value of the signal-tonoise distributions are:

- $\quad$ TOB thick sensors : $\mathrm{S} / \mathrm{N}=31-32$

- $\quad$ TIB/TID thin sensors : $\mathrm{S} / \mathrm{N}=25 / 29$

- $\quad$ TEC thin sensors : $\mathrm{S} / \mathrm{N}=27-30$

- $\quad$ TEC thick sensors : $\mathrm{S} / \mathrm{N}=31-36$

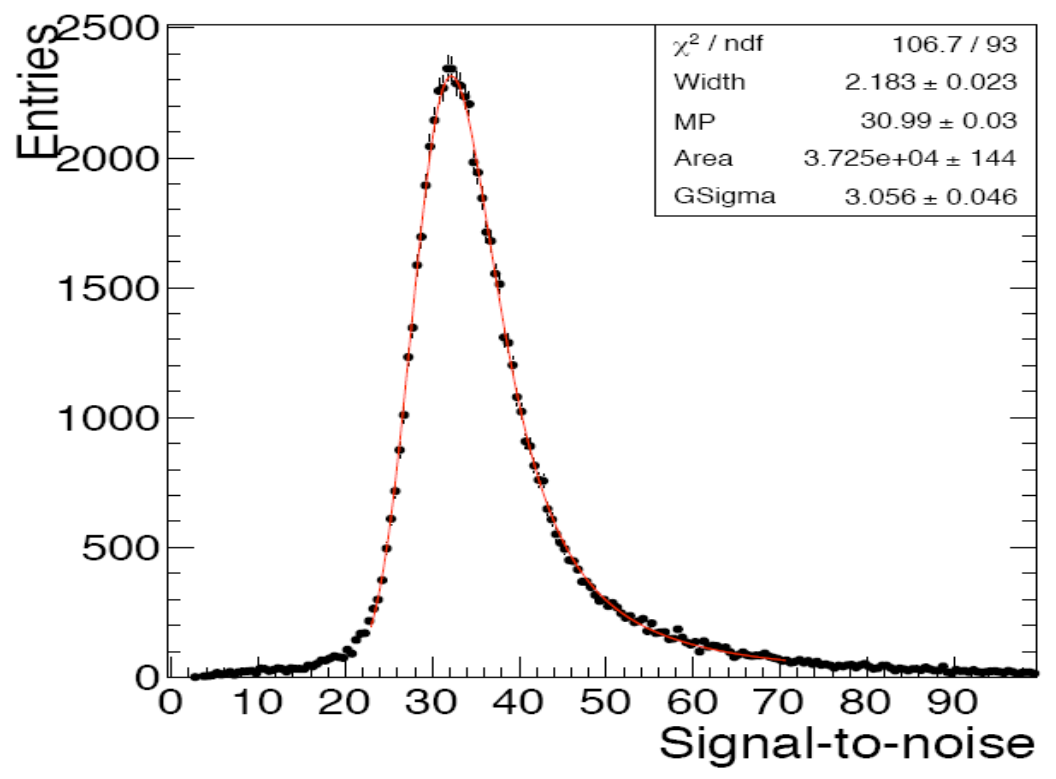

Fig.1. Signal to noise ratio in TOB. Landau distribution convoluted with Gaussian is used to fit the data. 
Preliminary measurements of signal to noise ratio in deconvolution mode of APV25 indicate that it scales as expected.

\section{Cross talk}

In CMS the silicon detectors signal is collected on implant strips, which are capacitively coupled to readout metallization strips. Due to the non-zero capacitive coupling to the neighboring readout strip there is a possibility for a signal to be induced on the neighboring channels. This effect is referred to as cross talk. The cross talk is best characterized using the so called "virgin raw data", where no thresholds are applied to the signal from strips, taken with magnetic field off. To suppress the charge sharing, only the tracks perpendicular to the sensor surface were selected and the amount of charge deposited on the neighboring strip was measured. Fig. 2 shows the charge ratio, where the denominator is the maximum strip charge of the cluster and the numerator is the charge on the strip adjacent to it. The data (points) are compared to the Monte Carlo simulation (histogram), where the cross talk is tuned to the measured value.

\section{Charge Ratio, TOB layers 1-4}

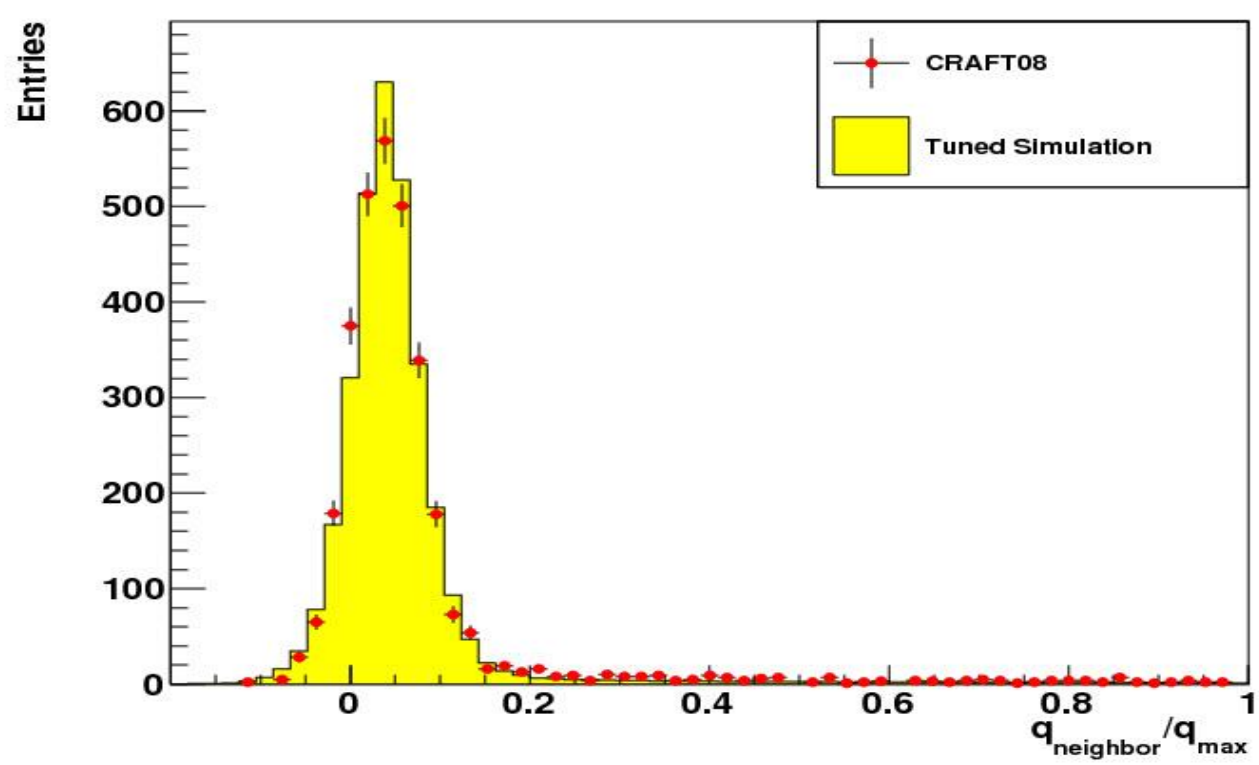

Fig 2. Charge ratio, where the denominator is the maximum strip charge of the cluster and the numerator is the charge on the strip adjacent to it. The data (points) are compared to the Monte Carlo simulation (histogram), where the cross talk is tuned to the measured value.

The amount of cross talk varies by layer, but it is typically below $3 \%$ in the APV25 peak mode. It is expected that in the deconvolution mode of the APV25 operation, better suited for collisions, the cross talk will be higher.

\section{Lorentz angle}

When the tracker is operated inside a magnetic field, electric charges produced by the passage of ionizing particles drift in crossed magnetic and electric fields. The direction 
of motion of these charges has an angle with respect to the normal to the sensor surface, or, in other words, with respect to the direction of their motion in absence of a magnetic field. This angle is commonly referred to as the Lorentz angle. It is proportional to the Hall mobility of the charge carriers, in this case holes, and the magnetic field. The ionization drift in magnetic field leads to a shift in the cluster centroid that needs to be corrected for the cluster position determination. As a result it also has a direct effect on the detector alignment using tracks. In addition, the cluster width is affected with an impact on the precision of the position measurement. Thus, it is important to determine the value of the Lorentz angle and introduce it in the simulation. One can determine the value of the Lorentz angle by studying the dependence of the cluster size, defined as the number of strips in a cluster on the track incidence angle (as shown in Fig. 3) [4]. The cluster width is expected to be at its minimum, when the track crosses the sensor with an angle equal to the Lorentz angle. The corresponding values of hole mobility are $-\mu_{\mathrm{H}}$ (TIB $300 \mu \mathrm{m} /$ TOB 500 sensors) $=0.018 / 0.023$.

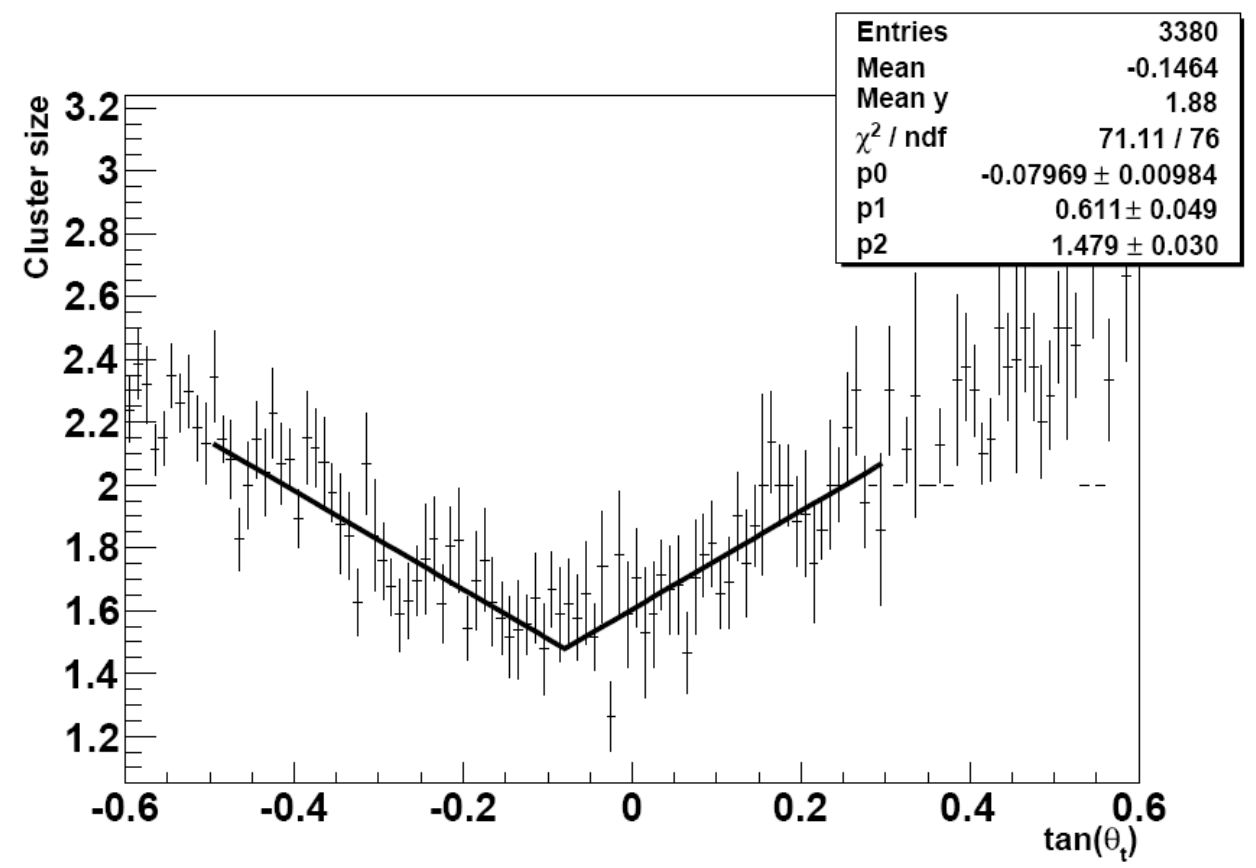

Fig 3. Profile of the cluster size vs tangent of the track incidence angle.

\section{Monte Carlo simulation tuning}

The CRAFT08 data set was also used to tune the Monte Carlo simulation to reproduce the data in an accurate way. Examples of distributions of cluster charge and detector occupancy are shown in Fig. 4. Only the random noise is generated in Monte Carlo simulation. A good agreement between data and simulation in the cluster multiplicity per event implies a low contribution from a possible coherent noise in the detector. 


\section{Conclusion}

The CMS collaboration has designed, built and installed a large scale silicon microstrip tracker. A multi-step commissioining process using cosmic ray muons proved that the device has a signal-to-noise ratio exceeding 25 and it is ready for the LHC collision data.
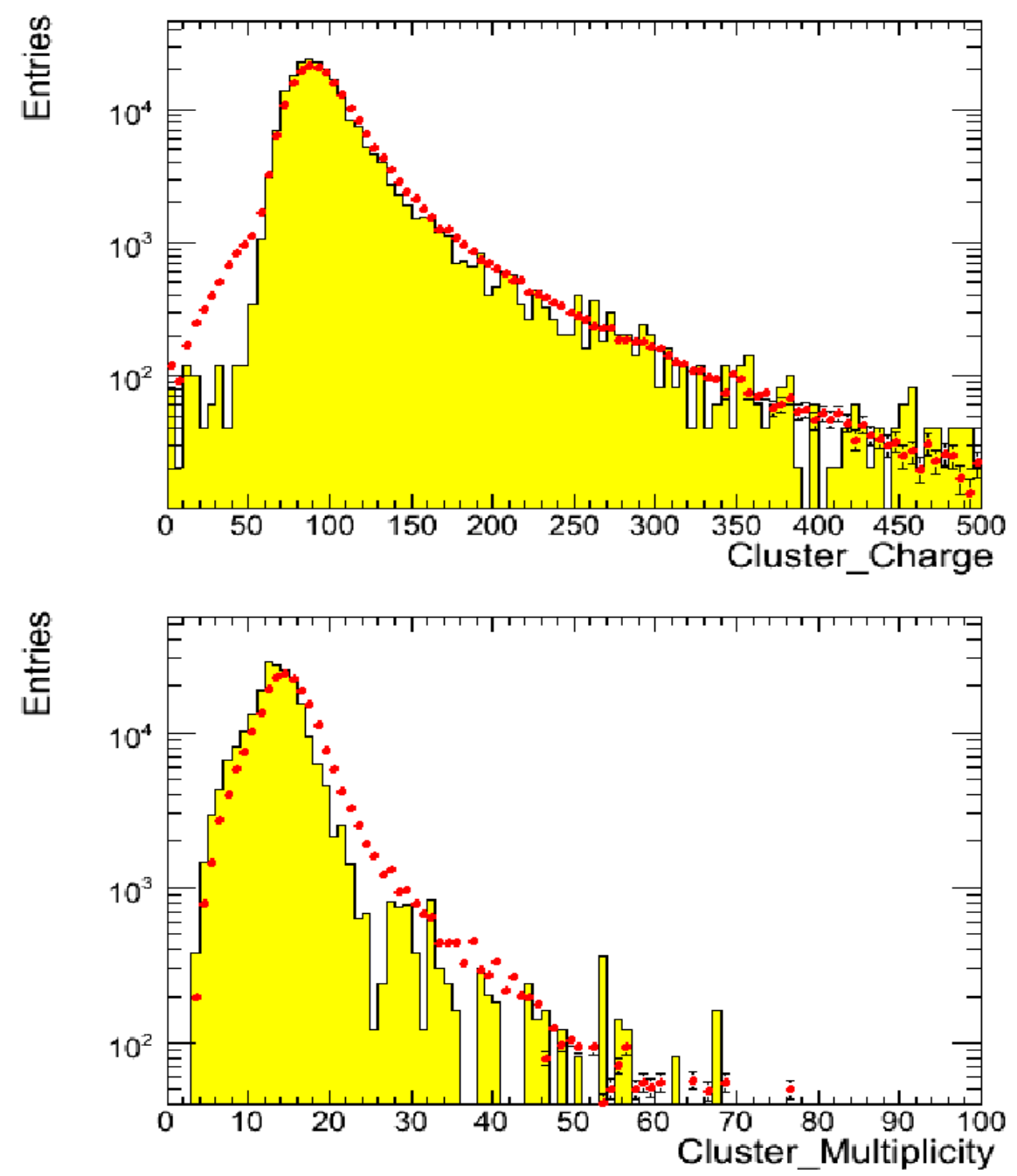

Fig 4.Comparison between data (points) and Monte Carlo simulation (histogram). Top plot: the cluster charge in ADC counts. Bottom plot: the cluster multiplicty per event, containing at least one reconstructed cosmic muon track.

\section{References}

1. The CMS collaboration, The CMS tracker: Addendum to the Technical Design Report, CERN-LHCC-2000-016.

2. S.N. Ahmed et al., "The DO RunIIa Silicon Microstrip Tracker", (to be submitted to Nucl. Inst. and Meth A). 
3. M.Raymond et al, "The CMS Tracker APV25 $0.25 \mathrm{~mm}$ CMOS Readout Chip", Proceedings of the 6th workshop on electronics for LHC experiments, CERN/LHCC/2000-041, 130-134.

4. D. Benedetti et al., Tracking and Alignment with the Silicon Strip Tracker at the CMS Magnet Test Cosmic Challenge, CERN-CMS-Note-2007-030.

5. H. Bichsel "Straggling in thin silicon detectors" Rev. Mod. Phys. 60, 663699

6. V. Ciulli, R. D'Alessandro, S. Frosali and C. Genta, Determination of the Lorentz Angle in Microstrip Silicon Detectors with Cosmic Muons, CERNCMS-Note-2008-006. 